\title{
Cellular phone dermatitis at an unusual site
}

\section{Osung Kwon, Yongwoo Choi, Hyun Chung, Joonsoo Park}

\author{
Department of Dermatology, School of Medicine, Catholic University of Daegu, Namgu, Daegu, South Korea
}

Corresponding author: Joonsoo Park, M.D., Ph.D., E-mail: ashkwon@naver.com

Sir,

Since Pazzaglia et al [1] reported cell phone dermatitis in 2000, a lot of cases and original articles were reported worldwide. Cellular phone dermatitis is a type of allergic contact dermatitis due to the direct contact with the metal from cellular phone, usually due to nickel. The typical locations of the lesions are ear, cheek, and periocular area [2]. Herein, we report a case of cellular phone dermatitis in unusual location, the buttock.

A 47-year-old woman presented with $1 \times 2 \mathrm{~cm}$ sized erythematous to brownish patch on her right buttock area (Fig. 1). The lesion started as small patch four months ago. She complained of severe itching and tingling sensation. She did not have any past history of skin disease and denied any allergic condition. The punch biopsy was performed on the lesion. On histopathologic examination, the lesion showed spongiosis of basal layer and lymphohistiocytic infiltration in papillary dermis (Fig. 2). On the next visit under the impression of contact dermatitis, the history was retaken carefully. She reported that she was working in a manufacturing plant and when she was working, she sweated a lot because of the high temperature. She also reported that she often contained her cellular phone in her right back pocket of pants. Patch test was performed using contact dermatitis kit (True test, Smartpractice demark aps, Hillerod, Denmark) and the result showed a 2-day + and 4-day ++ reaction on nickel sulphate (Fig. 3). She was diagnosed as allergic contact dermatitis due to nickel from cellular phone and the lesion got improved after antihistamine and topical steroid treatment.

As the worldwide use of cellular phone is increasing, cases of cellular phone dermatitis is also increasing [3].
Unlike the other cases, the location of lesion was buttock in this case and it was difficult to suspect the cellular phone dermatitis at first sight. In authors knowledge this is the first case of cellular phone dermatitis occurring on buttock. People often carry their cellular phone in the back pocket of their pants, so buttock also should be regarded as occurring lesion

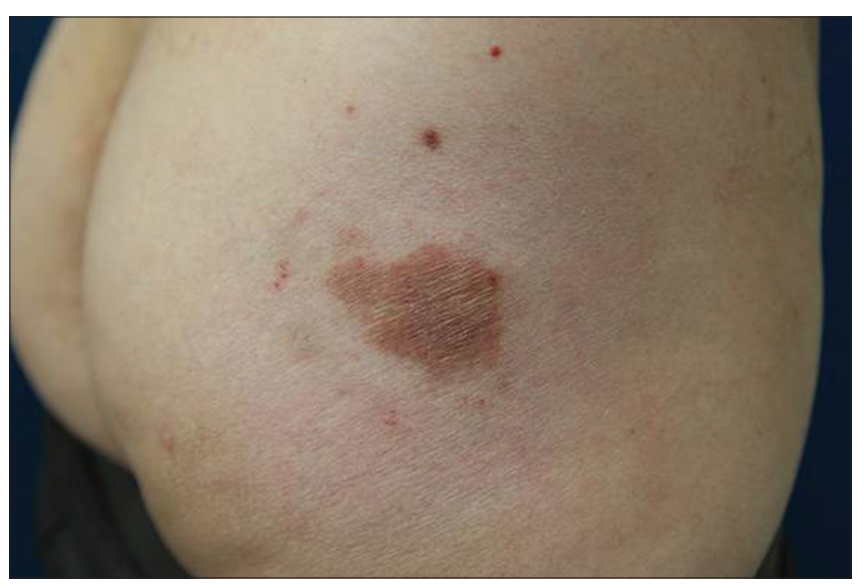

Figure 1: Erythematous to brownish patch on her right buttock area.

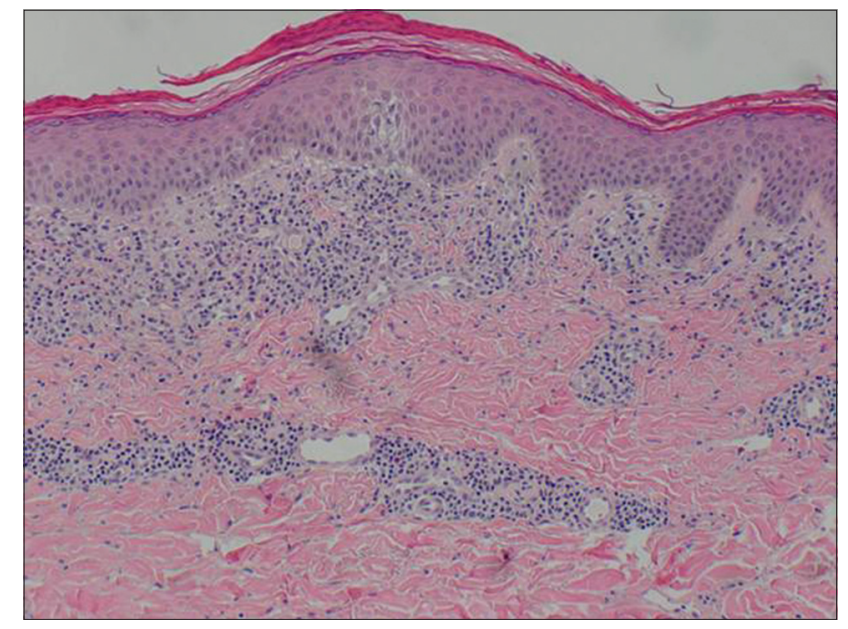

Figure 2: Histopathologic examination revealed sponiosis of basal layer and lymphohistiocytic infiltration in papillary dermis. (H\&E, X 100)

\footnotetext{
How to cite this article: Kwon O, Choi Y, Chung H, Park J. Cellular phone dermatitis at an unusual site. Our Dermatol Online. 2017;8(4):487-488. Submission: 16.03.2017; Acceptance: 10.08.2017 DOI: $10.7241 /$ ourd.20174.137
} 
www.odermatol.com

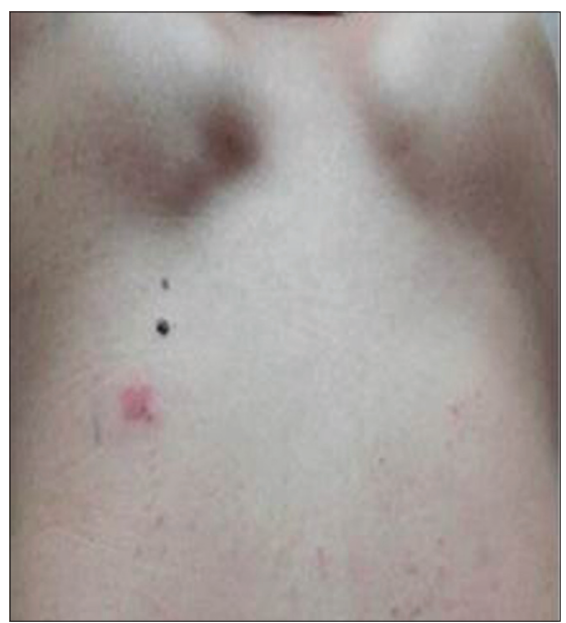

Figure 3: Three months after discontinuing of the drug shows improved skin lesion with noticeably regressed grouped skin colored papules leaving subtle hyperpigmentation.

of cellular phone dermatitis. Also when a patient is in condition of lots of sweating, it could precipitate the penetration of nickel to skin and increase the occurrence of contact dermatitis [4].
In conclusion, cellular phone dermatitis should be considered in the differential diagnosis of contact dermatitis of face, thigh, and buttock as well. If the cellular phone dermatitis is suspected patch test and dimethylglyoxime test may be helpful in establishing the diagnosis.

\section{REFERENCES}

1. Passaglia M, Lucente P, Vincenzi C, Tosti A. Contact dermatitis from nickel in mobile phones. Contact Dermatitis. 2000;42:362-3.

2. Guarneri F, Guarneri C, Cannavo SP. An unusual case of cell phone dermatitis. Contact Dermatitis. 2010;62:117.

3. Woehrl S, Jandl T, Stingl G, Kinaciyan T. Mobile telephone as new source for nickel dermatitis. Contact Dermatitis. 2007;56:113.

4. Livideanu C, Giordano-Labadie F, Paul C. Cellular phone addiction and allergic contact dermatitis to nickel. Contact Dermatitis. 2007;57:130-1.

Copyright by Osung Kwon, et al. This is an open-access article distributed under the terms of the Creative Commons Attribution License, which permits unrestricted use, distribution, and reproduction in any medium, provided the original author and source are credited.

Source of Support: Nil, Conflict of Interest: None declared. 